\title{
Mortality of patients with chronic obstructive pulmonary disease: a nationwide population- based cohort study
}

\author{
Seon Cheol Park ${ }^{1,2}$, Dong Wook Kim³ ${ }^{3}$ Eun Cheol Park ${ }^{4}$, Cheung Soo Shin ${ }^{5}$, Chin Kook Rhee ${ }^{6}$, \\ Young Ae Kang ${ }^{7}$, and Young Sam Kim ${ }^{7}$
}

\author{
${ }^{1}$ Division of Pulmonology, \\ Department of Internal Medicine, \\ National Health Insurance \\ Service Ilsan Hospital, Goyang; \\ ${ }^{2}$ Department of Medicine, Yonsei \\ University College of Medicine, \\ Seoul; ${ }^{3}$ Department of Policy \\ Research Affairs, National Health \\ Insurance Service Ilsan Hospital, \\ Goyang; Departments of ${ }^{4}$ Preventive \\ Medicine and ${ }^{5}$ Anesthesiology and \\ Pain Medicine, Yonsei University \\ College of Medicine, Seoul; ${ }^{6}$ Division \\ of Pulmonary, Allergy and Critical \\ Care Medicine, Department of \\ Internal Medicine, College of \\ Medicine, Seoul St. Mary's Hospital, \\ The Catholic University of Korea, \\ Seoul; ${ }^{7}$ Division of Pulmonology, \\ Department of Internal Medicine, \\ Yonsei University College of \\ Medicine, Seoul, Korea
}

Received: December 27, 2017 Revised : March 2, 2018 Accepted: May 4, 2018
Background/Aims: Chronic obstructive pulmonary disease (COPD) is a major cause of mortality in the world. There are no population-based studies on longterm mortality in COPD patients in Korea.

Methods: Using the large, population-based, National Health Insurance Service-National Sample Cohort (NHIS-NSC), we identified COPD patients using the International Classification of Disease-Tenth Revision (ICD-10) and prescription details in the NHIS-NSC during 2003 to 2013 . We analyzed the survival curves of COPD patients by sex, age, and cause of death.

Results: A total of 14,127 individuals older than 40 years were diagnosed with COPD. There were a total of 3,695 deaths $(26.2 \%)$ in COPD patients during the study period. The 5 -year mortality of COPD patients was $25.4 \%(29.9 \%$ in males and $19.1 \%$ in females). The mortality rate increased rapidly with age. The most common cause of death in COPD was chronic lower respiratory disease.

Conclusions: This study described long-term mortality in COPD patients in Korea. Higher mortality was observed in males, and it was closely related to age.

Keywords: Pulmonary disease, chronic obstructive; Mortality; Cause of death

\author{
Correspondence to \\ Young Sam Kim, M.D. \\ Division of Pulmonology, Department of Internal Medicine, Yonsei University College of \\ Medicine, 50 Yonsei-ro, Seodaemun-gu, Seoul 03722, Korea \\ Tel: +82-2-2228-1971, Fax: +82-2-393-6884, E-mail: ysamkim@yuhs.ac
}

\section{INTRODUCTION}

Chronic obstructive pulmonary disease (COPD), characterized by persistent respiratory symptoms and airflow limitation, is mainly caused by smoking or other environmental exposures and is a major cause of chronic morbidity and mortality in the world [1]. The burden of COPD is expected to increase in the coming decades due to an aging population and longer exposure to COPD risk factors such as smoking or air pollution [2]. COPD was the third leading cause of death worldwide in 2015, when 3.2 million people died from the disease [3].

Although many studies have described COPD mortality, there are no population-based studies on the long-term mortality of COPD in Korea. The purpose of this study was to analyze long-term mortality in COPD patients over 
10 years using a large population-based cohort.

\section{METHODS}

\section{Source of data}

Since 2000, the National Health Insurance Service (NHIS) in Korea has provided health insurance to nearly all people living in Korea. Consequently, a large amount of health-related data has accumulated in the NHIS database, leading to establishment of a research database. The target population consists of 46,605,433 individuals in the NHIS database system, from which a total of 1,025,340 participants were randomly sampled for a research database called the NHIS-National Sample Cohort (NSC). The NHIS-NSC included $2.2 \%$ of the total Korean population in 2002, following them for 12 years until 2013. We used this NHIS-NSC database to analyze mortality trends in COPD patients. This study was approved by the Institutional Review Board of National Health Insurance Service Ilsan Hospital (approval number: 2017-05-007). Written informed consent was waived by the board because data were de-identified and the study was retrospective in nature.

\section{Study population}

Because the NHIS database did not include spirometry data considered essential for the diagnosis of COPD, we identified COPD patients using the International Classification of Disease-Tenth Revision (ICD-10) and prescription details in the NHIS-NSC (Fig. 1). Similar to previous studies [4-6], COPD patients were identified by having all of the following criteria: older than 40 years; ICD-10 codes for COPD (J43-J44, except J430); and COPD medication use at least twice per year. COPD medications included long-acting muscarinic antagonists (LAMAs), long-acting beta-2 agonists (LABAs), inhaled corticosteroids (ICS), ICS plus LABA, short-active muscarinic antagonists (SAMAs), short-acting beta-2 agonists (SABAs), methylxanthines, systemic corticosteroids, and systemic beta agonists. We excluded COPD patients who were diagnosed in 2002, focusing on patients newly diagnosed with COPD from 2003 to 2013.

\section{Determinants of disease and demographic factors}

Demographic factors of COPD patients were analyzed

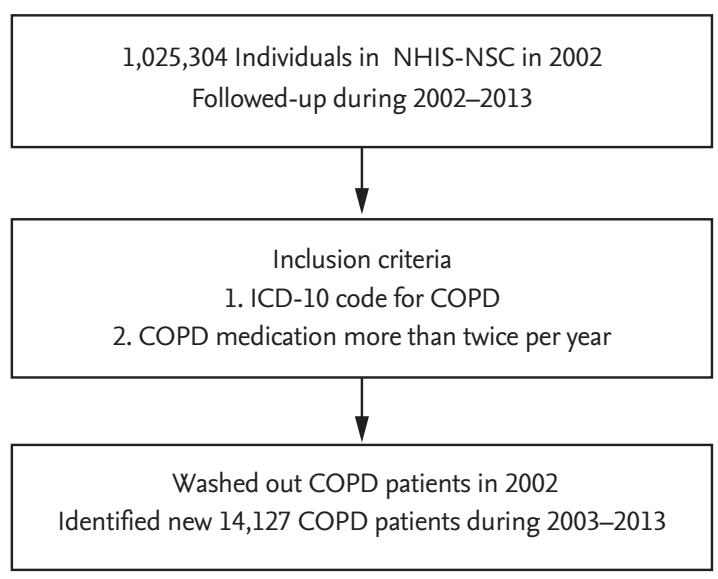

Figure 1. Flow chart of inclusion criteria for chronic obstructive pulmonary disease (COPD) identification. NHIS-NSC, National Health Insurance Service-National Sample Cohort; ICD10, International Classification of Disease-Tenth Revision.

at the time of COPD diagnosis. According to household income, socioeconomic status was divided into five categories. Region of residence was categorized to urban and rural area. Charlson comorbidity index was calculated as previously described [7].

\section{Mortality}

Patients were enrolled at the time of COPD diagnosis and followed until the end of the study period or death. The time of death was recorded by year and month. The immediate cause of death in the death certificate was used to classify the cause of death, which was divided into pneumonia (J12-J18), chronic lower airway disease (J40-J47), lung cancer ( $\left.\mathrm{C}_{34}\right)$, cardiovascular disease (I2O-I25, I30-52), cerebrovascular disease (I60-69), diabetes mellitus (E10-14), and hypertension (I10-15) according to the Korean Standard Classification of Disease and Cause of Death-Sixth revision.

\section{Statistical analysis}

Descriptive statistics were performed for all variables. Annual COPD visits by sex and age were calculated. First visits with COPD were deemed newly diagnosed COPD and used for the incidence analysis. A Kaplan-Meier analysis was used to calculate overall survival of COPD by sex and age. The causes of death in COPD patients were also analyzed. All statistical analyses were performed with SAS software version 9.4 (SAS Institute, Cary, NC, USA). 
Table 1. Baseline demographics of patients with chronic obstructive pulmonary disease

\begin{tabular}{|c|c|c|c|}
\hline Variable & Total $(\mathrm{n}=14,127)$ & Male $(\mathrm{n}=8,301)$ & Female $(n=5,826)$ \\
\hline \multicolumn{4}{|l|}{ Age, yr } \\
\hline $40-49$ & $946(6.7)$ & $495(6.0)$ & $451(7 \cdot 7)$ \\
\hline $50-59$ & $2,058(14.6)$ & $1,192(14.4)$ & $866(14.9)$ \\
\hline $60-69$ & $3,881(27.5)$ & $2,449(29 \cdot 5)$ & $1,432(24.6)$ \\
\hline $70-79$ & $4,670(33.1)$ & $2,832(34 \cdot 1)$ & $1,838(31.6)$ \\
\hline$\geq 80$ & $2,572(18.2)$ & $1,333(16.1)$ & $1,239(21.3)$ \\
\hline \multicolumn{4}{|c|}{ Household income } \\
\hline 1st quintile & $2,356(16.7)$ & $1,281(15 \cdot 4)$ & $1,075(18.5)$ \\
\hline 2nd quintile & $1,955(13.8)$ & $1,148(13.8)$ & $807(13.9)$ \\
\hline 3rd quintile & $2,300(16.3)$ & $1,384(16.7)$ & $916(15 \cdot 7)$ \\
\hline $4^{\text {th quintile }}$ & $2,927(20.7)$ & $1,784(21.5)$ & $1,143(19.6)$ \\
\hline $5^{\text {th }}$ quintile & $4,589(32.5)$ & $2,704(32.6)$ & $1,885(32.4)$ \\
\hline \multicolumn{4}{|c|}{ Region of residence } \\
\hline Urban & $11,393(80.6)$ & $6,733(81.1)$ & $4,660(80.0)$ \\
\hline Rural & $2,734(19.4)$ & $1,568(18.9)$ & $1,166(20.0)$ \\
\hline \multicolumn{4}{|c|}{ Charlson comorbidity index } \\
\hline o & $2,067(14.6)$ & $1,305(15.7)$ & $762(13.1)$ \\
\hline 1 & $3,826(27.1)$ & $2,212(26.7)$ & $1,614(27 \cdot 7)$ \\
\hline 2 & $3,493(24.7)$ & $2,055(24.8)$ & $1,438(24.7)$ \\
\hline$\geq 3$ & $4,741(33 \cdot 6)$ & $2,729(32.9)$ & $2,012(34 \cdot 5)$ \\
\hline Mortality & $3,695(26.2)$ & $2,525(30.4)$ & $1,170(20.1)$ \\
\hline
\end{tabular}

Values are presented as number (\%).

\section{RESULTS}

\section{Baseline demographics}

Table 1 shows the baseline demographics of COPD patients. A total of 14,127 individuals were diagnosed with COPD between 2003 and 2015 , and $58.8 \%$ of cases were males. Most patients were diagnosed with COPD between 70 and 79 years of age. COPD patients tended to have high socioeconomic status, urban residence, and a high Charlson comorbidity index. There was a total of 3,695 deaths (26.2\%) in COPD patients during the study period.

\section{Mortality rate}

The number of individuals who were newly diagnosed with COPD varied slightly during 2003 to 2015 , but the annual number did not show a significant trend toward increase or decrease. Table 2 shows the annual cumulative numbers of newly diagnosed COPD patients. The
Table 2. Annual newly diagnosed chronic obstructive pulmonary disease patients

\begin{tabular}{lcc}
\hline Year & No. (\%) & Cumulative no. (\%) \\
\hline 2003 & $1,346(9.5)$ & $1,346(9.5)$ \\
2004 & $1,280(9.1)$ & $2,626(18.6)$ \\
2005 & $1,188(8.4)$ & $3,814(27.0)$ \\
2006 & $1,164(8.2)$ & $4,978(35.2)$ \\
2007 & $1,217(8.6)$ & $6,195(43.9)$ \\
2008 & $1,269(9.0)$ & $7,464(52.8)$ \\
2009 & $1,336(9.5)$ & $8,800(62.3)$ \\
2010 & $1,263(8.9)$ & $10,063(71.2)$ \\
2011 & $1,321(9.4)$ & $11,384(80.6)$ \\
2012 & $1,538(10.9)$ & $12,922(91.5)$ \\
2013 & $1,205(8.5)$ & $14,127(100.0)$ \\
\hline
\end{tabular}

number of incidents was the lowest in 2006 at 1,164 and the highest in 2012 at 1,538 . The absolute number of an- 
nual deaths in COPD patients also increased. The mean follow-up duration of the whole population was $51.9 \pm$ 37.8 months. The mortality rates of all COPD patients 1 and 5 years after diagnosis were $9.5 \%$ and $25.4 \%$, respectively. The mortality rates differed significantly by sex and age (Figs. 2 and 3). The 5-year mortality rate in males was 1.5 times higher than in females (29.9\% vs. $19.1 \%)$. The mortality rate increased rapidly with age. The 5-year mortality rate grouped by age 40 to 49,50 to 59,60 to 69 ,

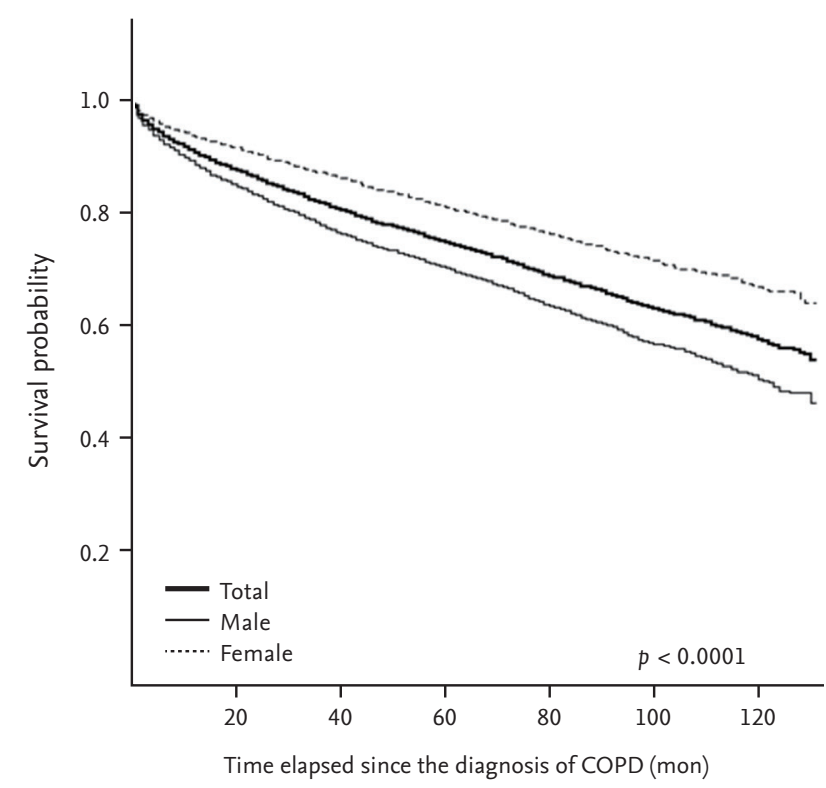

Figure 2. Survival curves of chronic obstructive pulmonary disease (COPD) patients by gender.
70 to 79 , and 80 to 89 was $3.1 \%, 7.8 \%, 14.1 \%, 30.4 \%$, and $55.1 \%$, respectively.

\section{Causes of death}

The most common causes of death in COPD were chronic lower respiratory disease (17.5\%), lung cancer (15.1\%), cardiovascular disease (8.3\%), and cerebrovascular disease (6.5\%) (Fig. 4). Lung cancer was the most common cause of death in men. Cardiovascular disease and

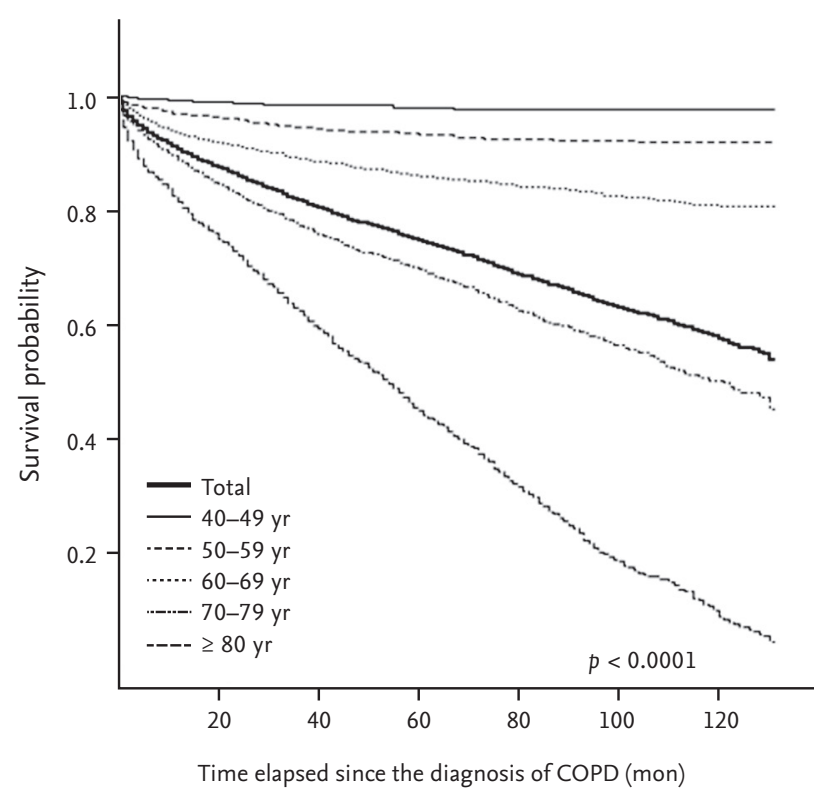

Figure 3. Survival curves of chronic obstructive pulmonary disease (COPD) patients by age.

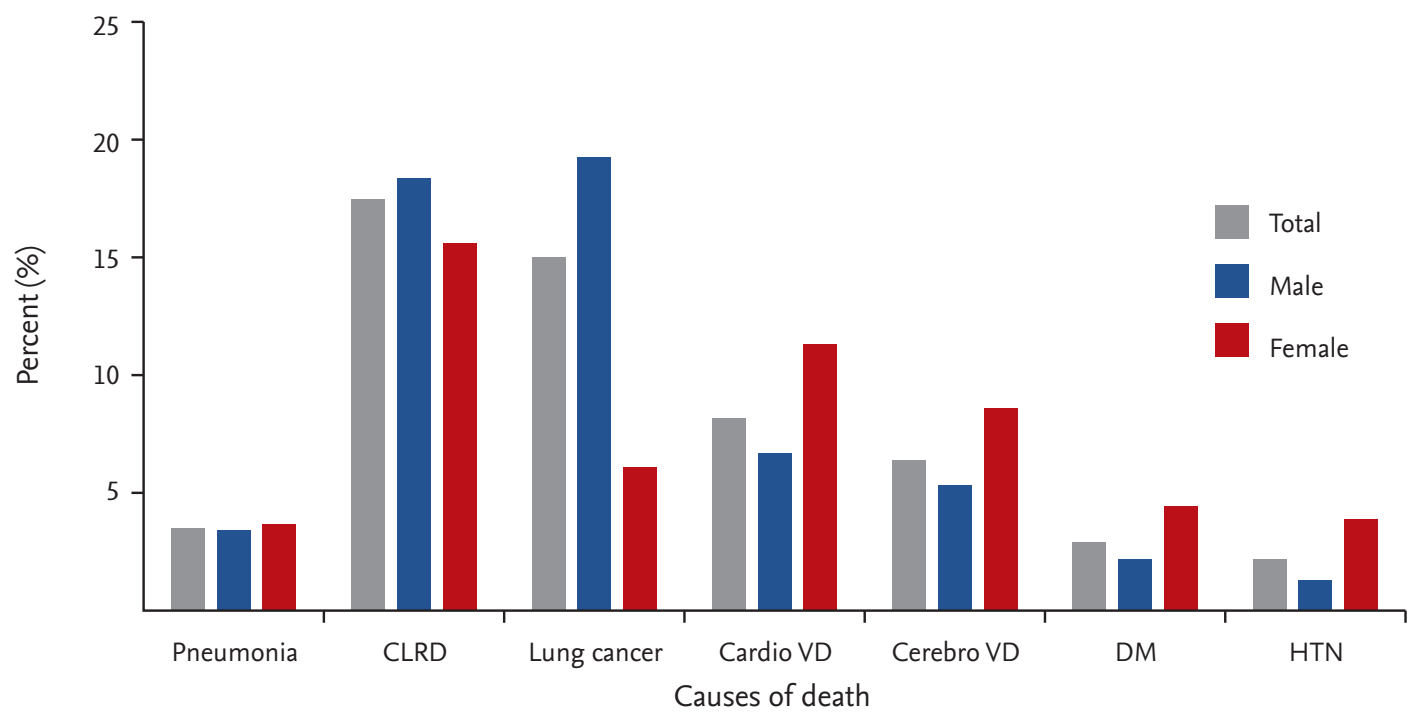

Figure 4. Causes of death in chronic obstructive pulmonary disease patients. CLRD, chronic lower respiratory disease; CardioVD, cardiovascular disease; CerebroVD, cerebrovascular disease; DM, diabetes mellitus; HTN, hypertension. 
cerebrovascular disease were common in women.

\section{DISCUSSION}

This is the first study to describe long-term mortality in COPD patients using a large population-based cohort in Korea. The 5-year mortality rate of COPD was $25.4 \%$. The mortality rate was higher in males (29.9\% vs. 19.1\%). The common causes of death in COPD were chronic lower respiratory disease, lung cancer, cardiovascular disease, and cerebrovascular disease.

Although the prevalence of COPD is well known in Korea, associated mortality has not previously been described. Globally, the prevalence of COPD is estimated as $11.7 \%$ [8]. The prevalence in Korea was $13.4 \%$ in those over 40 years of age in $2008,19.4 \%$ in males, and $7.9 \%$ in females [9]. Although the mortality rate of COPD has not previously been reported in Korea, annual statistics on causes of death in Korea have included the mortality rate of chronic lower airway disease [10]. Based on that report, the mortality rate of chronic lower airway disease has not change significantly over the past decade (from 14.4/100,000 in 2006 to 14.1/100,000 in 2014), and only $2.7 \%$ of all deaths in Korea are due to chronic lower airway disease. Chronic lower airway disease includes bronchitis, emphysema, COPD, asthma, and bronchiectasis according to the Korean Standard Classification of Disease and Cause of Death; accordingly, the reported mortality from pure COPD in Korea is very low.

Several studies have recently reported on the long-term mortality rate in COPD patients. Studies of hospitalized patients with acute exacerbation of COPD (AECOPD) report relatively high long-term mortality. One study has recounted a 1-year mortality of $26.2 \%$, while 5 -year mortality was $64.3 \%$ in patients admitted for AECOPD [11]. A similar study, which also analyzed patients with AECOPD, observed a long-term mortality (about 9 years) of COPD of $79 \%$ [12]. Population-based or outpatient-based studies have reported lower long-term COPD mortality than inpatient-based studies. One study in patients aged 65 and older patients found a COPD mortality rate of $56.9 \%$ after 12 years of follow-up [13]. Another study determined that the cumulative mortality of COPD over 7 years was $34.0 \%$, compared to $12.0 \%$ in the non-COPD population [14]. In subjects aged 65 to 100 years, COPD mortality at 5,10 , and 15 years was $32 \%, 62 \%$, and $75 \%$, respectively [15]. We found a relatively low long-term mortality rate of COPD (25.4\% 5-year mortality) because we included a younger population than other studies.

Although there are no studies of mortality trends from COPD in Korea, other countries have reported their statistics. In the United States, the age-adjusted COPD mortality rate has declined since 1999 [16]. The mortality rate was 63.7 per 100,000 people in 2011. However, the mortality rate in women has increased since 1999. In Europe, age-adjusted rates of COPD mortality decreased from 1994 to 2010 with a larger decrease in men and relatively stable rates in women $[17]$. The mortality rate per 100,000 people in 2010 was 61.33 in men and 25.15 in women. In China, the age-standardized COPD mortality rate decreased from 1990 to 2013 in both males and females [18]. The mortality rate in 2013 was 79.4 per 100,000 people. In Japan, age-adjusted COPD mortality decreased from 1950 to 2004 in both men and women [19]. The mortality rate per 100,000 people in 2004 was 19.7 in men and 4.3 in women. In all countries, the overall age-adjusted mortality from COPD has declined in recent years.

We found that chronic lower respiratory disease, lung cancer, and cardiovascular disease were the common causes of COPD mortality. This result was consistent with other studies [20-22]. The most frequent cause of death in COPD patients was deterioration of COPD. The Towards a Revolution in COPD Health (TORCH) study reported that $35 \%$ of COPD deaths were caused by pulmonary diseases [21]. However, only $17.5 \%$ of COPD patients in our study died from chronic lower respiratory disease. COPD might be underreported as a cause of death because COPD patients often die with other diseases. Previous studies have reported that one-third of COPD patients die from cardiovascular disease [21,23]. In fact, the mortality rate of chronic lower respiratory disease in Korea has been unchanged since 2006, while the mortality rate of COPD comorbidities such as pneumonia, lung cancer, and cardiovascular disease has increased [10].

The quality of death certificates could be the main problem with the COPD mortality analysis. Some studies suggest that COPD is underreported as a cause of death, which could lead to underestimation of the mortality burden from COPD. A study examining causes of death using data from the Copenhagen City Heart Study found that, among subjects with very severe COPD, only 
$24.9 \%$ had COPD listed as a cause of death [24]. The study also found that more than half of patients with very severe COPD did not have COPD mentioned on their death certificate. A similar outcome has been reported from a study in China, which found that a national vital registration system had $73.5 \%$ sensitivity for COPD as a cause of death [25]. Collectively, these findings suggest that estimates of COPD as a cause of death need to be reviewed carefully, and that death in COPD patients is closely associated with vascular disease mortality.

This study had limitations. First, we could not use spirometry data. We used only ICD-10 codes and medications for COPD diagnosis, which could result in selection bias. However, Korean NHIS only reimbursed the cost of inhaled long-acting bronchodilators when patients received spirometry and satisfied the criteria of airflow limitation. Therefore, by implication, physicians prescribed inhaled long-acting bronchodilators on the basis of spirometry. Second, individuals only took oral medications could be included as COPD patients. The main treatment of COPD is obviously inhalers. However, previous studies in Korea have showed that oral medications were prescribed frequently for COPD management, even more than inhalers [4,6,26]. Our working definition including oral agents as COPD medications has been utilized and validated in many previous studies [4-6]. Third, COPD patients without healthcare could not be included. Our results might not apply to all COPD patients in the general population, especially mild COPD patients without healthcare use. The male proportion of $58.5 \%$ in our study was lower than that of previous studies $[9,27]$. There is a possibility that healthcare use of men might be less than that of women $[28,29]$, and under-diagnosis of COPD might be more frequent in men. Similar to our study, previous COPD studies using Korean Health Insurance Review and Assessment Service database also reported relatively low male proportions [4-6].

In conclusion, this is the first population-based study that described long-term mortality in COPD patients in Korea. The 5-year mortality of COPD patients was about $25.4 \%$. Higher mortality was observed in males and the elderly. The 5 -year mortality rate in males was about 1.5 times higher than in females. The common causes of death in COPD were chronic lower respiratory disease and lung cancer.

\section{KEY MESSAGE}

1. This is the first population-based study that showed the long-term mortality of chronic obstructive pulmonary disease (COPD) in Korea.

2. The 5 -year mortality rate of COPD was about $25 \%$, and the mortality in males was higher than in females.

3. The most common cause of death in COPD was chronic lower respiratory disease.

\section{Conflict of interest}

No potential conflict of interest relevant to this article was reported.

\section{Acknowledgments}

This study used NHIS-NSC data (NHIS-2017-2-477) made by National Health Insurance Service (NHIS). The authors declare no conflict of interest with NHIS.

\section{REFERENCES}

1. Lopez AD, Shibuya K, Rao C, et al. Chronic obstructive pulmonary disease: current burden and future projections. Eur Respir J 2006;27:397-412.

2. Mathers CD, Loncar D. Projections of global mortality and burden of disease from 2002 to 2030. PLoS Med 2006;3:e442.

3. GBD 2015 Mortality and Causes of Death Collaborators. Global, regional, and national life expectancy, all-cause mortality, and cause-specific mortality for 249 causes of death, 1980-2015: a systematic analysis for the Global Burden of Disease Study 2015. Lancet 2016;388:1459-1544.

4. Kim J, Rhee CK, Yoo KH, et al. The health care burden of high grade chronic obstructive pulmonary disease in Korea: analysis of the Korean Health Insurance Review and Assessment Service data. Int J Chron Obstruct Pulmon Dis 2013;8:561-568.

5. Kim J, Kim K, Kim Y, et al. The association between inhaled long-acting bronchodilators and less in-hospital care in newly-diagnosed COPD patients. Respir Med 2014;108:153-161.

6. Rhee CK, Yoon $\mathrm{HK}$, Yoo $\mathrm{KH}$, et al. Medical utilization and cost in patients with overlap syndrome of chron- 
ic obstructive pulmonary disease and asthma. COPD 2014;11:163-170.

7. Charlson ME, Pompei P, Ales KL, MacKenzie CR. A new method of classifying prognostic comorbidity in longitudinal studies: development and validation. J Chronic Dis 1987;40:373-383.

8. Adeloye D, Chua S, Lee C, et al. Global and regional estimates of COPD prevalence: systematic review and meta-analysis. J Glob Health 2015;5:020415.

9. Yoo KH, Kim YS, Sheen SS, et al. Prevalence of chronic obstructive pulmonary disease in Korea: the fourth Korean National Health and Nutrition Examination Survey, 2008. Respirology 2011;16:659-665.

10. Statistics Korea. Annual report on the causes of death statistics, 2014 [Internet]. Daejeon (KR): Statistics Korea, c1996 [cited 2018 Aug 13]. Available from: http://kostat. go.kr/portal/korea/index.action.

11. Garcia-Sanz MT, Canive-Gomez JC, Senin-Rial L, et al. One-year and long-term mortality in patients hospitalized for chronic obstructive pulmonary disease. J Thorac Dis 2017;9:636-645.

12. Gudmundsson G, Ulrik CS, Gislason T, et al. Long-term survival in patients hospitalized for chronic obstructive pulmonary disease: a prospective observational study in the Nordic countries. Int J Chron Obstruct Pulmon Dis 2012;7:571-576.

13. Testa G, Cacciatore F, Bianco A, et al. Chronic obstructive pulmonary disease and long-term mortality in elderly subjects with chronic heart failure. Aging Clin Exp Res 2017;29:1157-1164.

14. Laforest L, Roche N, Devouassoux G, et al. Frequency of comorbidities in chronic obstructive pulmonary disease, and impact on all-cause mortality: a population-based cohort study. Respir Med 2016;117:33-39.

15. Sorino C, Pedone C, Scichilone N. Fifteen-year mortality of patients with asthma-COPD overlap syndrome. Eur J Intern Med 2016;34:72-77.

16. Ford ES. Trends in mortality from COPD among adults in the United States. Chest 2015;148:962-970.

17. Lopez-Campos JL, Ruiz-Ramos M, Soriano JB. Mortality trends in chronic obstructive pulmonary disease in Europe, 1994-2010: a joinpoint regression analysis. Lancet Respir Med 2014;2:54-62.
18. Yin $\mathrm{P}$, Wang $\mathrm{H}$, Vos $\mathrm{T}$, et al. A subnational analysis of mortality and prevalence of COPD in China from 1990 to 2013: findings from the Global Burden of Disease Study 2013. Chest 2016;150:1269-1280.

19. Pham TM, Ozasa K, Kubo T, et al. Age-period-cohort analysis of chronic obstructive pulmonary disease mortality in Japan, 1950-2004. J Epidemiol 2012;22:302-307.

20. Anthonisen NR, Connett JE, Enright PL, Manfreda J; Lung Health Study Research Group. Hospitalizations and mortality in the Lung Health Study. Am J Respir Crit Care Med 2002;166:333-339.

21. Calverley PM, Anderson JA, Celli B, et al. Salmeterol and fluticasone propionate and survival in chronic obstructive pulmonary disease. N Engl J Med 2007;356:775-789.

22. Sin DD, Anthonisen NR, Soriano JB, Agusti AG. Mortality in COPD: role of comorbidities. Eur Respir J 2006;28:1245-1257.

23. Anthonisen NR, Skeans MA, Wise RA, et al. The effects of a smoking cessation intervention on 14.5-year mortality: a randomized clinical trial. Ann Intern Med 2005;142:233-239.

24. Jensen HH, Godtfredsen NS, Lange P, Vestbo J. Potential misclassification of causes of death from COPD. Eur Respir J 2006;28:781-785.

25. Rao C, Yang G, Hu J, Ma J, Xia W, Lopez AD. Validation of cause-of-death statistics in urban China. Int J Epidemiol 2007;36:642-651.

26. Kim C, Yoo KH, Rhee CK, et al. Health care use and economic burden of patients with diagnosed chronic obstructive pulmonary disease in Korea. Int J Tuberc Lung Dis 2014;18:737-743.

27. Kim DS, Kim YS, Jung KS, et al. Prevalence of chronic obstructive pulmonary disease in Korea: a population-based spirometry survey. Am J Respir Crit Care Med 2005;172:842847.

28. Suh DC, Lau H, Pokras SM, Choi IS, Valiyeva E. Sex differences in ambulatory visits for chronic obstructive pulmonary disease, based on the National Ambulatory Medical Care Survey and the National Hospital Ambulatory Medical Care Survey from 1995 to 2004. Respir Care 2008;53:1461-1469.

29. Endevelt R, Baron-Epel O, Viner A, Heymann AD. Socioeconomic status and gender affects utilization of medical nutrition therapy. Diabetes Res Clin Pract 2013;101:20-27. 